\title{
Electrochemical Oxidation and Cleavage of Proteins with On-line Mass Spectrometric Detection: Development of an Instrumental Alternative to Enzymatic Protein Digestion
}

\author{
Hjalmar P. Permentier and Andries P. Bruins \\ Center for Pharmacy, University of Groningen, Groningen, The Netherlands
}

An electrochemical flow cell coupled on-line to a mass spectrometer is used to oxidize a range of proteins. Oxidation of tyrosine and tryptophan can give rise to peptide bond cleavage at their C-terminal side. This suggests the possible use of electrochemistry as an alternative protein digestion method. For the small proteins insulin and $\alpha$-lactalbumin ( 6 and $14 \mathrm{kD}$ ) almost all potential sites are cleaved, while for the largest successfully tested protein (carbonic anhydrase, $29 \mathrm{kD}$ ) 7 of the 15 available sites were specifically cleaved. Several proteins did not produce peptides upon electrochemical oxidation, possibly due to problems with accessibility of tyrosine and tryptophan residues, or to competing oxidation reactions. Peptides were generally not the major oxidation products: non-cleavage oxidation products observed as protein mass $+\mathrm{n} \times 16 \mathrm{Da}$, presumably by oxidation of tyrosine, tryptophan, cysteine and methionine, account for the major fraction of protein oxidation products. Nevertheless the amount and variety of cleavage products at the present conditions shows good prospects for further improvement of the system. The efficient protein oxidation also allows the use of the EC-MS system as a tool to study protein oxidation reactions in general. The preconditioning and life history and/or age of the electrochemical cell was relevant to the solvent and sample conditions needed for efficient oxidative cleavage as opposed to other oxidation reactions. (J Am Soc Mass Spectrom 2004, 15, 1707-1716) ( 2004 American Society for Mass Spectrometry

$\mathrm{P}$ eptide mapping, or peptide mass fingerprinting, is a fast and powerful method for identification of unknown proteins. It relies on residue-specific cleavage of proteins into a set of smaller peptides with masses that are predictable from the amino acid sequence of the parent protein. The set of peptide masses is compared with a protein database to find the bestmatching protein. Further confirmation of identify can be achieved by (partial) sequencing of individual peptides by MS/MS techniques.

Protein cleavage by hydrolysis of the peptide bonds is traditionally achieved in two ways: by proteolytic enzymes or by chemical reagents. Enzymes and chemicals can be specific for one or a few amino acid residues or sequences, or they can be largely or completely aspecific, depending on their reaction mechanism. Specific, and therefore predictable, cleavage of the protein is essential for peptide mapping. The most widely used

Published online October 26, 2004

Address reprint requests to Dr. A. P. Bruins, Center for Pharmacy, University of Groningen, A. Deusinglaan 1, 9713 AV Groningen, The Netherlands. E-mail: a.p.bruins@farm.rug.nl proteolytic enzyme in peptide mapping studies is trypsin, which specifically hydrolyzes proteins at the Cterminal side of arginine and lysine, when the Cterminal residue is not proline. $\mathrm{CNBr}$ is a commonly used chemical compound that specifically cleaves proteins, in this case at the C-terminal side of methionine [1].

A possible alternative for enzymatic and chemical protein digestion that has received very little attention is electrochemistry. Electrochemical oxidation of amino acids readily occurs at the aromatic tyrosine $(\mathrm{Y})$, tryptophan $(\mathrm{W})$, and histidine $(\mathrm{H})$ and sulfur-containing cysteine $(\mathrm{C})$, and methionine $(\mathrm{M})[2,3]$. When these amino acids are present in peptides or proteins, the oxidized intermediates can react with adjacent parts of the molecule. We have recently shown this can result in specific cleavage of the peptide bond C-terminal to tyrosine [4]. An intermediate phenoxonium ion can react with the carbonyl group at the C-terminal side (Figure 1, top). Hydrolysis of the peptide bond produces an $\mathrm{N}$-terminal peptide with the tyrosine residue modified to a stable spirodienone-lactone group, and an unmodified C-terminal peptide. The electrochemical oxidation of the tyrosine side chain also generates the 

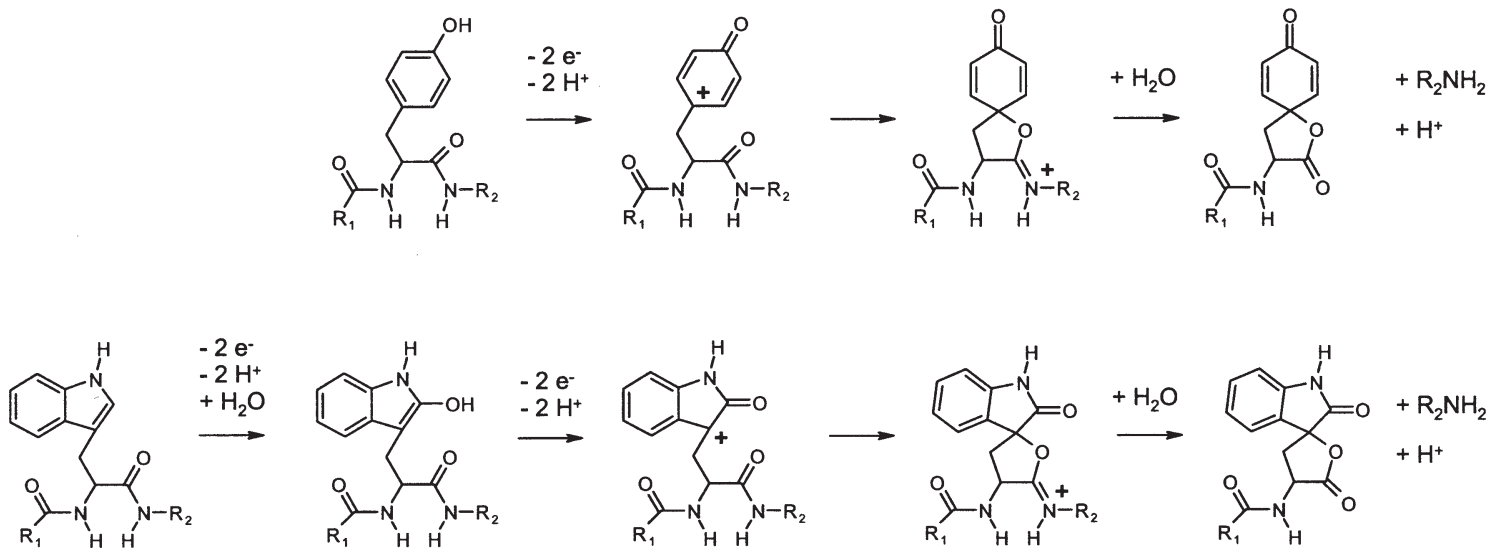

Figure 1. Proposed reaction mechanism for electrochemical oxidation and cleavage at tyrosine (top) and tryptophan (bottom). $\mathrm{R}_{1}$ and $\mathrm{R}_{2}$ are the parts of the protein $\mathrm{N}$-terminal and C-terminal, respectively, to tyrosine and tryptophan.

cyclohexadienone, catechol and quinone non-cleavage oxidation products, as concluded from the observed mass changes of the oxidized tyrosine residue [4].

Electrochemical cleavage of peptides at tyrosine had been described only a few times in the past 40 years [5-7]. An analogous cleavage reaction is possible Cterminal to tryptophan (Figure 1, bottom). Tryptophan is easily hydroxylated upon oxidation, most likely at position 2 of the indole group as indicated in Figure 1. Subsequently, hydroxytryptophan can be oxidized again and induce $\mathrm{C}$-terminal cleavage analogous to tyrosine. This reaction has been described by MacDonald and Roscoe [7] and by Walton and co-workers [8]. The latter is the only paper to date that reports electrochemical cleavage of a protein (hen egg white lysozyme); the other authors have used small (di)peptides.

Electrochemical cleavage reactions are very similar to cleavage by chemical oxidation by brominating or iodinating agents, on which the proposed mechanisms are based: tyrosine is specifically cleaved at the Cterminus by e.g., N-bromosuccinimide [9], while BNPSskatole is specific for tryptophan [1].

Electrochemical oxidation has several advantages over chemical oxidation. There is no need to remove reagents, and an electrochemical flow cell can be readily coupled on-line to a mass spectrometer, allowing fast and real-time analysis of oxidation reactions. MS and MS/MS provide powerful ways of characterizing the oxidation products. Incorporation of an HPLC separation step between the electrochemical cell and the mass spectrometer (EC-LC-MS) is possible to facilitate the analysis of complex product mixtures. Coupled EC-MS techniques have only been developed in the last decade and have many possible applications, e.g., the study and mimicking of in vivo oxidation and metabolism of natural compounds and drugs, and enhancement of ionization efficiency of analytes for MS detection [1013].

Protein oxidation by metabolic or environmental agents has become an important field of study in the last few years. With recent advances in MS techniques detailed studies of the extent and location of these oxidations are now possible [14]. Mimicking in vivo protein oxidation could help to understand the mechanisms and produce oxidized proteins for further research. Chemical oxidation is often used to this effect, but electrochemistry could become a useful alternative. Proteins with nitrated tyrosine residues have been prepared by electrochemical means [15]. In addition, the extent and location of protein oxidation gives an indication of accessibility of amino acids in proteins [16, 17], similar to H/D exchange experiments.

In the present study, a range of proteins is subjected to oxidizing potentials in an electrochemical flow cell and the resulting products are analyzed on-line by mass spectrometry. Peptide products are further characterized and identified by MS/MS. Separation of the various oxidation products by HPLC, with a coupled ECLC-MS system allows for even better analysis. The susceptibility of various proteins to electro-oxidative cleavage and the extent of cleavage are discussed, and the dependence on experimental conditions, including potential, solvent composition and cell history are stressed. The prospect for a fast, alternative system for protein digestion is demonstrated.

\section{Experimental}

\section{Chemicals}

Angiotensin I (human, synthetic), trypsinogen (bovine pancreas), ribonuclease A (RNase I, bovine pancreas type XII-A), $\alpha$-lactalbumin (bovine milk), carbonic anhydrase (bovine erythrocytes), lysozyme (chicken egg white), apomyoglobin (horse skeletal muscle), iodoacetamide, and dithiothreitol were purchased from Sigma (Zwijndrecht, The Netherlands). Neuropeptide Y (porcine) was obtained from Saxon Biochemicals (Hannover, Germany). Insulin (human, recombinant) was 
obtained as Actrapid solution from Novo Nordisk (Copenhagen, Denmark). Serum albumin (HSA, purified from human blood) was a kind gift from J. Eriksson (AstraZeneca, Sweden).

Acetonitrile (gradient grade) and formic acid (analytical grade) was obtained from Merck (Amsterdam, The Netherlands). Water was purified by a Maxima Ultrapure water system (ELGA, High Wycombe, Bucks, UK).

\section{Sample Preparation}

Protein solutions were prepared in 50/50/0.1 (vol/vol/ vol) acetonitrile/water/formic acid to a protein concentration of 5 or $10 \mu \mathrm{M}$. Additional formic acid was added to a final solvent composition of 47.5/47.5/5 ( $\mathrm{vol} / \mathrm{vol} /$ vol) acetonitrile/water/formic acid immediately before the electrochemistry experiments, to prevent acid hydrolysis or formylation of the proteins during storage.

Carbonic anhydrase and $\alpha$-lactalbumin were purified with a Microcon YM-10 centrifuge filter (Millipore, Etten-Leur, The Netherlands) which has a $10 \mathrm{kD}$ mass cut-off.

Human insulin was obtained as a $4 \mathrm{mg} / \mathrm{mL}$ solution (Actrapid, see above) containing several salts and additives. The solution was $70 \times$ diluted in 50/50/0.1 (vol/ $\mathrm{vol} / \mathrm{vol}$ ) acetonitrile/water/formic acid to obtain an insulin concentration of $10 \mu \mathrm{M}$. The solution may still contain appreciable amounts of salts $(\mathrm{ZnCl}, \mathrm{HCl}$ and $\mathrm{NaOH}$ are reported), which may function as supporting electrolytes. No significant salt adduct formation was observed, however.

For reduction and alkylation, lysozyme was dissolved to $100 \mu \mathrm{M}$ in water; dithiothreitol was added to a concentration of $2 \mathrm{mM}$ and incubated for $30 \mathrm{~min}$ at $60{ }^{\circ} \mathrm{C}$, followed by addition of iodoacetamide to a concentration of $2 \mathrm{mM}$ and incubation for $30 \mathrm{~min}$ at room temperature in the dark.

\section{Instrumentation}

A Brownlee microgradient system (Brownlee Labs, Santa Clara, CA) delivered an isocratic flow of 2-50 $\mu \mathrm{L} / \mathrm{min}$ to a Coulochem 5020 electrochemical guard cell or a Coulochem 5021 conditioning cell (ESA Inc., Bedford, MA). The working electrode is made from porous graphite and all reported cell potentials are versus a palladium reference electrode. The solvent consisted of $50 \%$ acetonitrile, $50 \%$ water, and $0.1 \%$ formic acid ( $\mathrm{pH}$ 2.8). For the experiments described in this paper no electrolytes other than formic acid were added to the solvent system. Samples were injected into a $100 \mu \mathrm{L}$ loop on a Rheodyne 7725 injector (Rheodyne, Rohnert Park, CA) directly in front of the cell.

Control of the cell potential of the 5020 guard cell was done by a home-made potentiostat that was in turn controlled by a MacLab system with Chart 3.5.7 software (AD Instruments, Castle Hill, NSW, Australia). The potential and current through the cell were contin- uously recorded by the MacLab system. The 5021 conditioning cell was controlled by an ESA Coulochem III electrochemical detector system with a single channel cable (p/n 70-4770, ESA), which allowed programming of the potential of the conditioning cell as if it were an analytical cell. The output current was recorded by the MacLab system as described above. For the described experiments, both set-ups have the same functionality with respect to potential control. In both cases, a constant potential was applied to the cell; this was switched on for several minutes before sample injection in order to equilibrate the electrode.

For EC-LC-MS the cell effluent was diluted with a make-up liquid flow consisting of $0.1 \%$ formic acid in water to an acetonitrile concentration of $5 \%$ or less. The combined flow was led through a $1.0 \mathrm{~mL}$ loop in a Rheodyne 7010 switch valve. After collecting the complete diluted sample plug in the loop, the valve was switched to the inject position: a gradient HPLC system (Series 200 micro LC pumps, Perkin Elmer, Norwalk, $\mathrm{CT})$ pumped through the loop onto a $150 \times 1.0 \mathrm{~mm}$ reversed phase HPLC column (218MS5115 Vydac, Hesperia, CA) with a guard column. The solvents were $0.1 \%$ formic acid in water (Solvent A) and $0.1 \%$ formic acid in acetonitrile (Solvent B). After 5 min of equilibration at $10 \%$ Solvent B, a 40 min gradient to $50 \%$ Solvent $\mathrm{B}$ was programmed, followed by $5 \mathrm{~min}$ of flushing at $90 \%$ Solvent B and re-equilibration at $10 \%$ Solvent B.

The cell outlet or the HPLC column effluent was connected to the TurboIonSpray source of an API3000 mass spectrometer (MDS-Sciex, Concord, Ontario, Canada). The turbo heat gun was only used in the LC-MS experiments, at $350{ }^{\circ} \mathrm{C}$. Full scan mass spectra of the cell or column effluent were continuously recorded, with step size 1 . The orifice voltage was set at $20-30 \mathrm{~V}$ to prevent up-front fragmentation of the peptides. Product ion spectra were obtained by CID MS/MS, with nitrogen as collision gas and step size 1 .

\section{Results and Discussion}

\section{Electrochemical Cleavage of Proteins}

Electrochemical cleavage of peptides C-terminal to tyrosine residues has been described recently [4]. This phenomenon was studied further with a number of larger peptides and proteins of various sizes and compositions as listed in Table 1 . The main goal of the experiments was to assess if, first of all, the proteins could be oxidized efficiently, and if oxidative cleavage takes place to a sufficient extent to make the method useful for protein digestion and subsequent peptide analysis or peptide mapping. The results of the oxidative cleavage are summarized in Table 1 and details of the observed peptides are listed in Table 2. All experiments were performed with a constant potential of 0.8 or $1.0 \mathrm{~V}$, in the presence of $5 \%$ formic acid and at a flow rate of $2 \mu \mathrm{L} / \mathrm{min}$ (see Cell Conditions section for more details). Of the nine proteins tested lysozyme, trypsino- 
Table 1. Proteins subjected to electrochemical oxidation, with their composition and the extent of electrochemical cleavage

\begin{tabular}{|c|c|c|c|c|c|c|c|}
\hline & $\begin{array}{l}\text { Swiss-Prot } \\
\text { accession } \\
\text { number }\end{array}$ & MW (kD) & $\mathrm{C}$ & $\mathrm{M}$ & Y & W & Peptides \\
\hline Neuropeptide Y & P01304 & 4.3 & 0 & 0 & $5 / 2$ & 0 & $5 / 2$ \\
\hline Insulin & P01308 & 5.8 & 6 & 0 & $4 / 4$ & 0 & $4 / 3$ \\
\hline RNase I & P00656 & 13.7 & 8 & 4 & $6 / 1$ & 0 & $4 / 1$ \\
\hline$\alpha$-Lactalbumin & P00711 & 14.2 & 8 & 1 & $4 / 3$ & $4 / 3$ & $7 / 4$ \\
\hline Lysozyme & P00698 & 14.3 & 8 & 2 & & & \\
\hline with SS bonds & & & & & $3 / 0$ & $6 / 0$ & $8 / 0$ \\
\hline without SS bonds & & & & & $3 / 2$ & $6 / 5$ & $10 / 4$ \\
\hline Apomyoglobin & P02188 & 17.0 & 0 & 2 & $2 / 1$ & $2 / 2$ & $5 / 3$ \\
\hline Trypsinogen & P00760 & 24.0 & 12 & 2 & $10 / 0$ & $4 / 0$ & $9 / 0$ \\
\hline Carbonic anhydrase & P00921 & 28.5 & 0 & 3 & $8 / 5$ & $7 / 2$ & $16 / 4$ \\
\hline Serum albumin & P02768 & 66.5 & 35 & 6 & $18 / 0$ & $1 / 0$ & $15 / 0$ \\
\hline
\end{tabular}

The protein molecular weights, number of oxidizable residues, and number of potential peptides are based on the amino acid sequences obtained from the Swiss-Prot database. All cysteines form disulfide bonds, except for one cysteine in serum albumin. The numbers before and after the slash for $\mathrm{Y}$ and $\mathrm{W}$ represent the number of residues present in the protein, and the number for which cleavage has been observed, respectively. The numbers before and after the slash in the "peptides" column indicate the predicted number of peptides (some linked by disulfide bridges), and the number of peptides observed upon electrochemical oxidation, respectively. Peptides with missed cleavages overlapping with observed completely cleaved peptides were omitted for simplicity.

gen and serum albumin did not produce peptides upon oxidation under these standard conditions, which were not optimized further for individual proteins. Reduction of disulfide bonds did result in cleavage products for lysozyme. The other six proteins showed partial or almost complete digestion. Of the potential peptides expected after specific cleavage, some are very small (a single $\mathrm{Y}$ or $\mathrm{W}$ residue) or very large $(>5 \mathrm{kD})$. As shown in Table 2, several large peptides could still be observed, but the ones smaller than three amino acids were not detected.

Figure 2 shows the mass spectra of $\alpha$-lactalbumin with and without oxidation. The top panel shows the spectrum measured at a cell potential of $0 \mathrm{~V}$, with the reconstructed protein spectrum shown in the inset. The average protein mass is calculated to be $14,186 \mathrm{Da}$, which corresponds with the first peak in the reconstructed spectrum. The other peaks have masses of about 100 Da higher, probably due to adducts of phosphoric acid. In the spectra measured at cell potentials of 0.8 and $1.2 \mathrm{~V}$ (Figure 2 middle and bottom panels) the multiply charged protein peaks became increasingly broader and shifted to higher $\mathrm{m} / \mathrm{z}$. The reconstructed protein peaks in the insets show a number of peaks spaced at about $16 \mathrm{Da}$ from the first peak at about $14,186 \mathrm{Da}$.

In addition to the changes in the protein peak pattern, a number of peaks in the lower mass range between $\mathrm{m} / \mathrm{z} 400$ and 1000 appear, most clearly in the 0.8 $\mathrm{V}$ spectrum (Figure 2). These peaks could be assigned to specifically cleaved peptides of $\alpha$-lactalbumin. Below $\mathrm{m} / \mathrm{z} 400$ many unidentified background peaks were observed, that were either produced by oxidation or induced by the increased formic acid concentration in the sample plug. To reduce the background peaks resulting from the sample itself, the spectrum measured at $0 \mathrm{~V}$ was subtracted from the $0.8 \mathrm{~V}$ spectrum (Figure 3 bottom). The peptides resulting from electrochemical cleavage have been indicated by numbers that refer to Figure 3 (top), which shows the sequence and disulfide bonds of $\alpha$-lactalbumin. Peaks labeled 2, 4, and 5 in Figure 3 have been subjected to MS/MS to confirm the identity the peptides. The MS/MS spectrum of Peptide 5 is shown in the inset of Figure 3. The observed fragments are consistent with the expected sequence of GLFQINNKIW, with $\mathrm{W}$ having a mass increase of $14 \mathrm{Da}$ (see Figure 1). Peaks at $m / z 561$ and 701 did not produce an adequate MS/MS spectrum, but they correspond to a multiply charged peptide of $2800 \mathrm{Da}$, consistent with the mass of Peptide 1 (Table 2). Peaks at $\mathrm{m} / \mathrm{z} 978$ and 1022 have been labeled tentatively as the 5 times charged Peptides 6 and $6+7$, respectively. They are not listed in Table 2 because of the lack of confidence in identification. No other peptides with missed cleavages were observed for $\alpha$-lactalbumin.

The number of peptides for $\alpha$-lactalbumin is still limited and most can be recognized easily from the mass spectrum, as shown in Figure 3. This will not always be the case, especially with larger proteins with many more $\mathrm{Y}$ and $\mathrm{W}$ residues. As is usual for tryptic peptide mixtures, LC-MS can be performed to separate the oxidation products for better identification. A complication for the present system is that the high acetonitrile concentration in the solvent $(50 \%)$ is not compatible with retention of peptides on a reversed phase HPLC-column. Therefore the cell effluent was combined with a make-up flow of water with $0.1 \%$ formic acid. This combined flow was then collected in a large loop or trapped on a guard column, before switching onto a reversed phase column with a gradient HPLCsystem. To avoid these complications and delays imposed by the current solvent conditions, an effort should be made to achieve oxidation at reduced acetonitrile concentrations, so that the cell effluent can be loaded directly onto the column.

To confirm the presence of the expected peptides in 
Table 2. Observed peptide products of electrochemically oxidized proteins

\begin{tabular}{|c|c|c|}
\hline Protein with amino acid sequence & Observed peptide & $\begin{array}{c}\text { Monoisotopic } \\
\text { mass (Da) }\end{array}$ \\
\hline \multicolumn{3}{|l|}{ Neuropeptide $Y$} \\
\hline YPSKPDNPGE DAPAEDLARY YSALRHYINL ITRQRY & $\begin{array}{l}\text { Y20-Y27 } \\
128-Y 36\end{array}$ & $\begin{array}{c}906.5^{\mathrm{a}} \\
1173.7\end{array}$ \\
\hline Insulin & & \\
\hline A-chain: GIVEQCCTSI CSLYQLENYC N & $A(G 1-Y 14)+B(F 1-Y 16)$ & 3339.6 \\
\hline B-chain: FVNQHLCGSH LVEALYLVCG ERGFFYTPKT & $\begin{array}{l}\text { A (Q15-Y19) } \\
B(T 27-T 30)\end{array}$ & $\begin{array}{l}663.3 \\
445.3\end{array}$ \\
\hline \multicolumn{3}{|l|}{ RNase I } \\
\hline KETAAAKFER QHMDSSTSAA SSSNYCNQMM KSRNLTKDRC & V116-V124 & 969.5 \\
\hline KPVNTFVHES LADVQAVCSQ KNVACKNGQT NCYQSYSTMS & & \\
\hline $\begin{array}{l}\text { ITDCRETGSS KYPNCAYKTT QANKHIIVAC EGNPYVPVHF } \\
\text { DASV }\end{array}$ & & \\
\hline \multicolumn{3}{|l|}{$\alpha$-Lactalbumin } \\
\hline EQLTKCEVFR ELKDLKGYGG VSLPEWVCTT FHTSGYDTQA & $(E 1-Y 18)+(L 118-L 123)$ & $2798.5^{c}$ \\
\hline IVQNNDSTEY GLFQINNKIW CKDDQNPHSS NICNISCDKF & G19-W26 & 857.4 \\
\hline LDDDLTDDIM CVKKILDKVG INYWLAHKAL CSEKLDQWLC & D37-Y50 & 1594.7 \\
\hline EKL & G51-W60 & 1245.7 \\
\hline \multicolumn{3}{|l|}{ Apomyoglobin } \\
\hline GLSDGEWQQV LNVWGKVEAD IAGHGQEVLI RLFTGHPETL & G1-W7 & $776.3^{\mathrm{b}}$ \\
\hline EKFDKFKHLK TEAEMKASED LKKHGTVVLT ALGGILKKKG & Q8-W14 & $899.5^{\mathrm{b}}$ \\
\hline HHEAELKPLA QSHATKHKIP IKYLEFISDA IIHVLHSKHP & K147-G153 & $777.4^{\mathrm{b}}$ \\
\hline GDFGADAQGA MTKALELFRN DIAAKYKELG FQG & & \\
\hline \multicolumn{3}{|l|}{ Carbonic anhydrase } \\
\hline SHHWGYGKHN GPEHWHKDFP IANGERQSPV DIDTKAVVQD & D70-Y87 & 1919.9 \\
\hline PALKPLALVY GEATSRRMVN NGHSFNVEYD DSQDKAVLKD & G127-Y189 & $4159.2^{c}$ \\
\hline GPLTGTYRLV QFHFHWGSSD DQGSEHTVDR KKYAAELHLV & P193-W207 & 1610.9 \\
\hline HWNTKYGDFG TAAQQPDGLA VVGVFLKVGD ANPALQKVLD & I208-W243 & $6541.4^{c}$ \\
\hline ALDSIKTKGK STDFPNFDPG SLLPNVLDYW TYPGSLTTPP & & \\
\hline LLESVTWIVL KEPISVSSQQ MLKFRTLNFN AEGEPELLML & & \\
\hline ANWRPAQPLK NRQVRGFPK & & \\
\hline \multicolumn{3}{|l|}{ Lysozyme (reduced and alkylated with iodoacetamide) } \\
\hline KVFGRCELAA AMKRHGLDNY RGYSLGNWVC AAKFESNFNT & S24-W28 & 589.3 \\
\hline QATNRNTDGS TDYGILQINS RWWCNDGRTP GSRNLCNIPC & G54-W62 & 1099.6 \\
\hline SALLSSDITA SVNCAKKIVS DGNGMNAWVA WRNRCKGTDV & V109-W111 & 388.2 \\
\hline QAWIRGCRL & I124-L129 & $773.4^{\mathrm{d}}$ \\
\hline
\end{tabular}

The monoisotopic masses of the peptides take into account disulfide bonds and modification of the $\mathrm{C}$-terminal $\mathrm{Y}$ and $\mathrm{W}$ residues. The latter have masses of 161.1 and 200.1 Da, respectively.

a peptide with 1 missed cleavage;

${ }^{b}$ Only detected with the old electrochemical cell (see text);

cidentification based on mass only, not confirmed with MS/MS

dincludes 1 alkylated cysteine residue.

the mixture of oxidation products of $\alpha$-lactalbumin, LC-MS was performed on-line with the electrochemical cell. The resultant LC-MS chromatogram is shown in Figure 4 . The identified peptides are again numbered according to Figure 3. Peaks 561 and 701 elute in a single peak, confirming the presence of the $2800 \mathrm{Da}$ peptide 1, consisting of two parts linked by a disulfide bond.

Two large peaks were present in the LC-MS chromatogram, that could not be assigned to the expected peptides. Their masses of 524 and $2245 \mathrm{Da}$ were not present in the mass spectrum shown in Figure 3. It is not clear whether these peaks are due to additional breakdown of peptides or the protein during LC separation, or that they result from background compounds (fragmentation again was inconclusive).

The broad peak eluting between 65 and $70 \mathrm{~min}$ resulted from the non-cleaved oxidized protein (see below). The sharp peak at $69.5 \mathrm{~min}$ contained the remaining unoxidized protein. EC-LC-MS analysis of the protein without applying a cell potential resulted in just a large peak at the same retention time (not shown). The broad shape of the oxidized protein peak suggests that it is heterogeneous, with respect to number and position of oxidized residues.

The spectra of oxidized carbonic anhydrase and RNase I shown in Figure 5 illustrate the very different cleavage patterns obtained with different proteins under the same experimental conditions. RNase I produces only a single peptide peak, but at a high intensity. This peptide corresponds to the C-terminal part of RNase I, which may be expected to be easily accessible for oxidation. Oxidation of carbonic anhydrase gives rise to several peptides widely distributed over the 


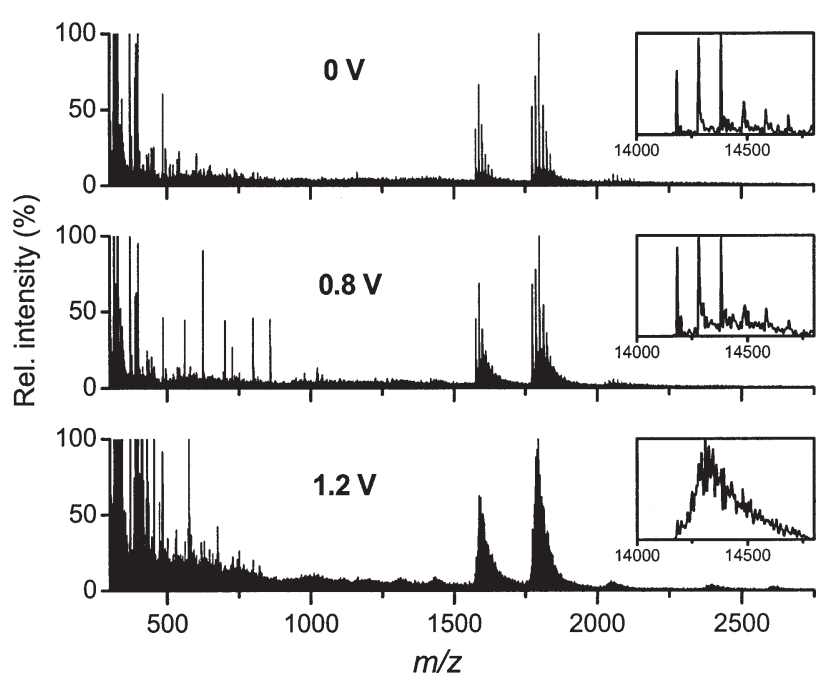

Figure 2. Mass spectra of $\alpha$-lactalbumin without electrochemical oxidation (cell potential of $0 \mathrm{~V}$, top panel), at a cell potential of 0.8 $\mathrm{V}$ (middle), and at $1.2 \mathrm{~V}$ (bottom). Flow rate was $2 \mu \mathrm{L} / \mathrm{min}$, and $5 \%$ formic acid was present in the sample. The insets show the reconstructed protein spectra.

protein. Seven cleavage events have to occur to produce the four observed peptides: only two peptides were adjacent in the protein (3 and 4 in Figure 5). Carbonic anhydrase does not contain any disulfide bonds, probably facilitating access to the whole protein chain.

The presence of disulfide bonds was expected to be major cause of lack of cleavage products for some proteins. This was addressed by comparing the oxidation products of lysozyme with and without breaking of the disulfide bonds. When disulfide bonds are still present, only the multiply charged protein peaks are observed (Figure 6 top). When the cysteines are reduced and alkylated with iodoacetamide, however, several peptides could be observed (Figure 6 bottom). The cleavages sites adjacent to the four observed peptides (listed in Table 2) cover 6 of the 8 tyrosines and tryptophans. The larger peptides are either not recovered from the cell or have insufficient signal intensity. Generally signal intensity is greatly impaired by residual iodoacetamide, that could not be removed sufficiently by either dialysis or C18 ZipTip purification.

Peptides resulting from aspecific electrochemical cleavage (at residues other than $\mathrm{Y}$ and $\mathrm{W}$ ) have not been identified so far. It cannot be excluded that some aspecific cleavage occurs: there are a number of peaks in the MS and LC-MS spectra of e.g., $\alpha$-lactalbumin that had an intensity that was too low for MS/MS analysis. These peaks may also include specifically cleaved peptides present at low concentration. Peptides with missed cleavages were observed much less frequently than completely cleaved ones. As with tryptic digests, not all peptides will be observed due to ionization problems. Higher concentrations or amounts of protein could be used to get higher intensity product peaks, but this will most likely change the distribution of reaction products, which is not desirable.
In addition to peaks related to the injected protein sample, a number of peaks with high intensity were usually observed that could not be readily identified. Their MS/MS spectra did not look like typical peptide fragmentation spectra. As expected, more non-peptide oxidation products were observed at higher than at lower potentials (see Figure 2). The identity of these and other "contamination peaks" is of interest, since it will allow fuller understanding of the range of reactions occurring at the electrode with or without protein. These undesirable peaks can then be excluded from further analysis and their formation can be prevented by adjustment of the solvent conditions.

\section{Oxidation Without Cleavage}

The major oxidation products of the proteins used in this study are usually not the peptides but the uncleaved oxidized proteins (see Figures 2 and 5). The reconstructed protein spectra (e.g., Figure 2, insets) always show successive mass increases of about $16 \mathrm{Da}$. We have not yet investigated these oxidized proteins in more detail, but most likely oxidation of cysteine and methionine to sulfoxides and sulfones and non-cleavage oxidation of tyrosine and tryptophan all contribute to the mass increases. For tyrosine-containing peptides, non-cleavage $\mathrm{M}-2, \mathrm{M}+14$, and $\mathrm{M}+16$ Da oxidation products were shown to be modified at the tyrosine residues [4].

Interestingly, disulfide bonds present in cleavage products do not appear to be readily oxidized. The 3344 Da peptide of insulin is not further oxidized at $0.8 \mathrm{~V}$, while at 1.0 and $1.2 \mathrm{~V}$, small peaks of $\mathrm{M}+16$ and $\mathrm{M}+$ $32 \mathrm{Da}$ appear (results not shown). These mass increases may be assigned to oxidation of disulfide bonds, since no other oxidizable residues are present in the peptide. The same absence of disulfide bond oxidation at $0.8 \mathrm{~V}$ was observed for the 2800 Da peptide 1 of $\alpha$-lactalbumin (see above).

\section{Cell Conditions}

In the course of these experiments, we encountered an important issue regarding the reproducibility of the results obtained with the electrochemical cells used for earlier experiments [4]. These experiments were performed with ESA 5020 guard cells that had been used for a number of years for electrochemical detection purposes. After they were no longer sensitive enough for detection, they had been used for a several years for the electrochemical oxidation of a wide variety of small molecules $[11,13]$ that may have slowly and irreversibly coated the working electrode. Reversible adsorption of peptide samples, leading to severe tailing of injection plugs, could be attributed to the contamination of the electrode surface. Generally, contaminated electrodes are cleaned by washing and polishing. Washing with $2 \mathrm{M}$ nitric acid was needed to remove the revers- 

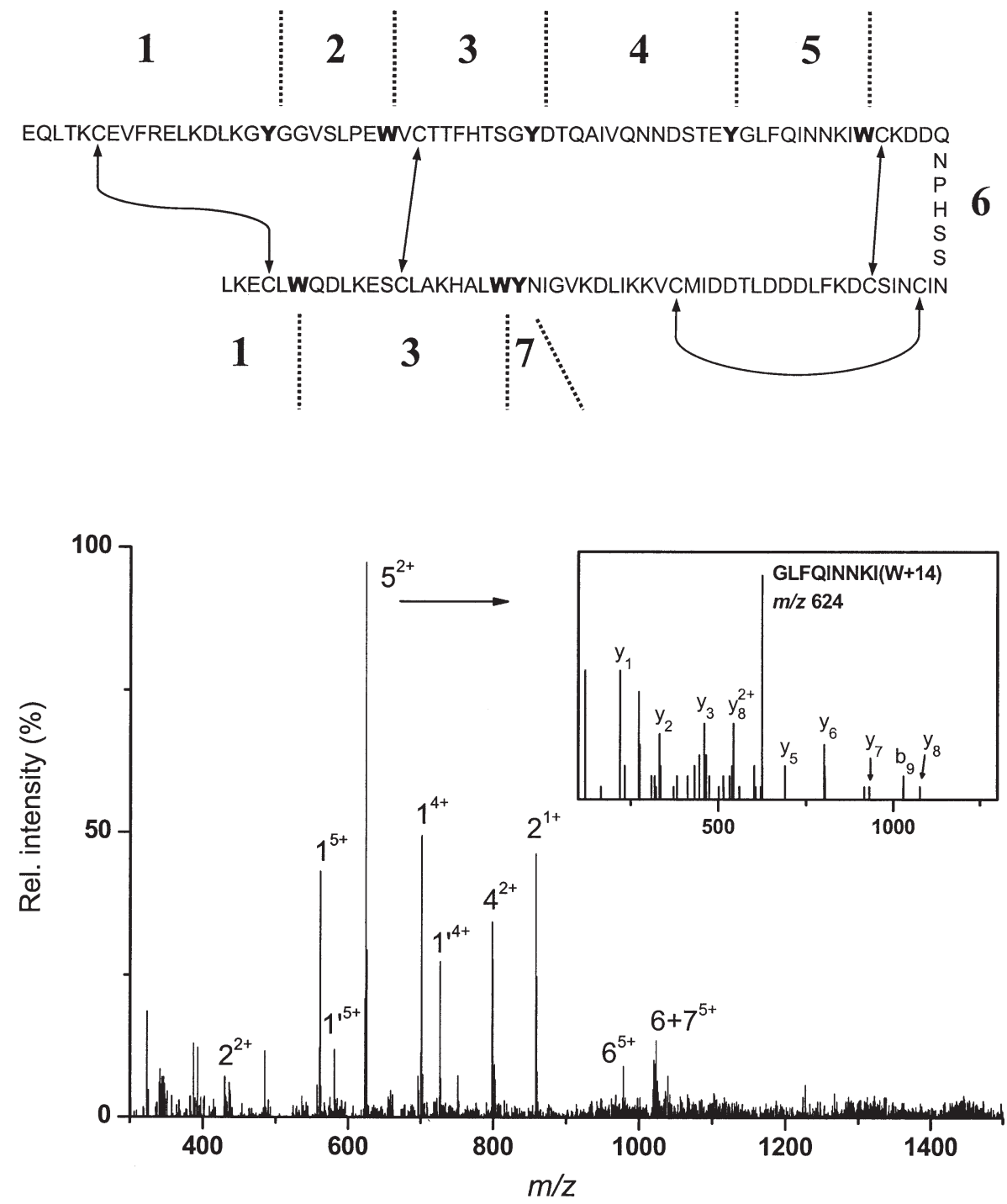

Figure 3. Top: Structure of $\alpha$-lactalbumin. The tyrosine and tryptophan residues are shown in boldface; disulfide bonds are indicated with arrows. The peptides produced by complete cleavage at $\mathrm{Y}$ and $\mathrm{W}$ residues are separated by dotted lines and numbered. Bottom: Mass spectrum of $\alpha$-lactalbumin oxidized at $0.8 \mathrm{~V}$, after subtraction of the spectrum measured at $0 \mathrm{~V}$ (see Figure 2 ). The peaks labels refer to the peptide numbers in Figure 4. Inset: product ion spectrum obtained by collision-induced dissociation MS/MS of the oxidation product observed at $\mathrm{m} / \mathrm{z} 624(2+)$. The fragment ions corresponding to the amino acid sequence GLFQINNKI $(\mathrm{W}+14)$ are labeled.

ibly bound oxidation products [4]. Polishing of the porous graphite electrode is not possible.

To overcome the adsorption problems, a new, unused cell (ESA 5021 conditioning cell) with the same type of porous graphite electrode was used to continue the experiments. Much less tailing of sample plugs was observed, indicating less adsorption to the electrode. Unexpectedly however, the ratio of observed oxidation products in the new cell, with angiotensin I as a test peptide [4], was rather different than with the old cell under the same sample and solvent conditions (Figure $7 \mathrm{a}$ and $\mathrm{b}$ ). Peptide dimerization yield was significantly increased at the expense of peptide cleavage (Figure $7 \mathrm{~b})$. Dimerization was only occasionally observed with the old cell [4], although formation of dityrosine by

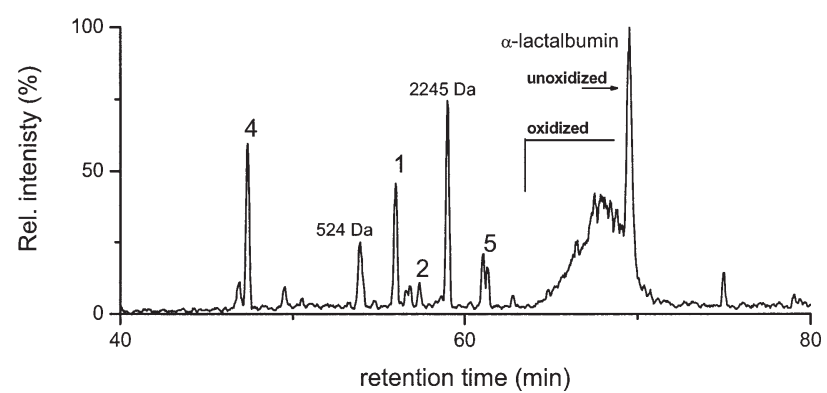

Figure 4. LC-MS base peak chromatogram $(\mathrm{m} / \mathrm{z} 400-3000)$ of $\alpha$-lactalbumin after on-line electrochemical oxidation at $0.8 \mathrm{~V}$. The peptide peaks are labeled according to Figure 4; the peaks labeled $524 \mathrm{Da}$ and $2245 \mathrm{Da}$ could not be identified as peptides formed by cleavage of $\alpha$-lactalbumin. 

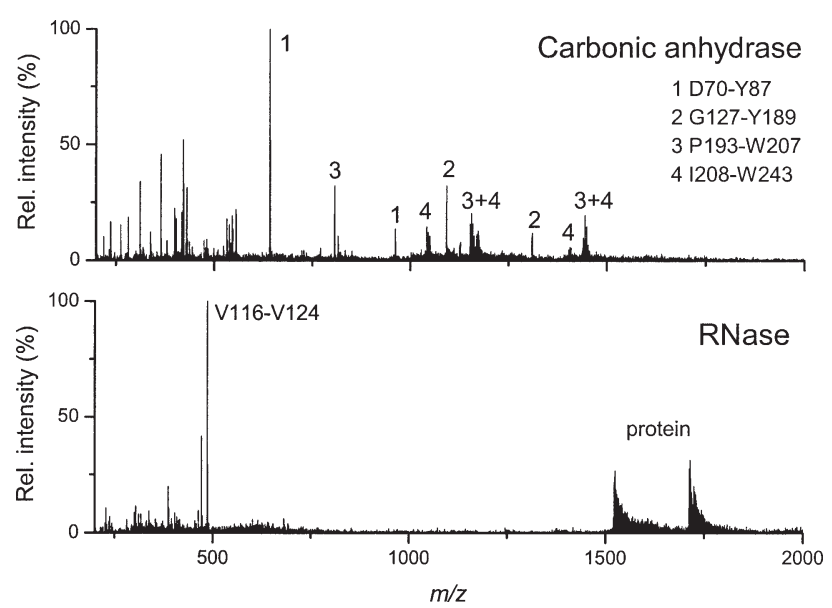

Figure 5. Mass spectra of carbonic anhydrase (top panel) and RNase I (bottom) after oxidation at $1.0 \mathrm{~V}$; the other experimental conditions were the same as for Figure 2. The masses of the labeled peptides are listed in Table 2.

cross-linking of tyrosine residues easily occurs upon chemical oxidation [18], and analogous electrochemical cross-linking is common for compounds containing a phenol group [19].

A number of variables were changed, including sample concentration, flow rate, type and concentration of the supporting electrolyte, but no single factor improved cleavage yield to the level achieved with the old cell. A comparable cleavage yield of angiotensin I was finally obtained by simultaneous changing of two factors: a significantly lower flow rate $(2 \mu \mathrm{L} / \mathrm{min}$ instead of $10-50 \mu \mathrm{L} / \mathrm{min}$ ) combined with addition of $5 \%$ (vol/ vol) formic acid to the sample (Figure 7c). The lower flow rate increases the residence time of the sample in the cell and therefore the contact time with the electrode. This may now be comparable to the old cell,

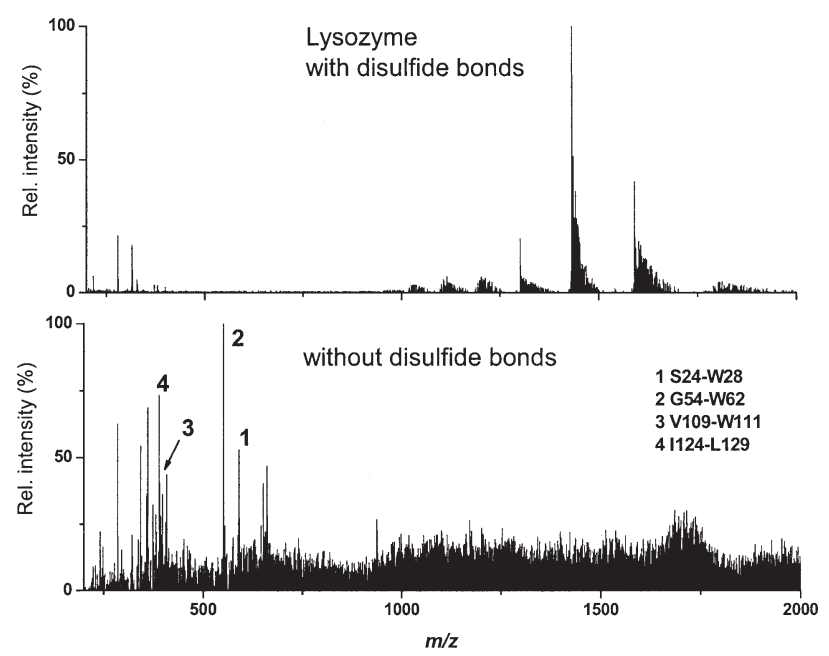

Figure 6. Mass spectra of lysozyme after oxidation at $1.0 \mathrm{~V}$ and a flow rate of $2 \mu \mathrm{L} / \mathrm{min}$, without prior breaking of disulfide bonds (top) and after reducing of disulfide bonds and alkylation of cysteines with iodoacetamide (bottom). The masses of the labeled peptides are listed in Table 2.

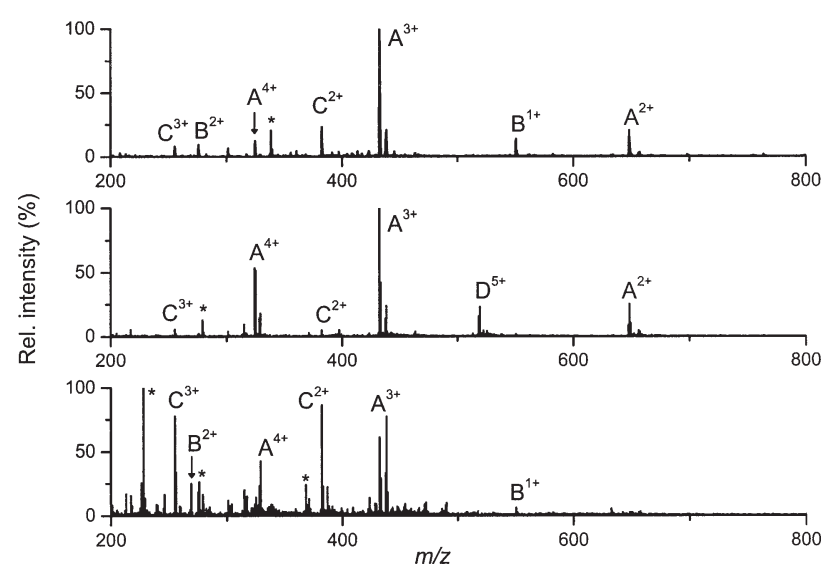

Figure 7. Comparison of electrochemical oxidation of angiotensin I under different conditions. Top panel: background-corrected spectrum obtained with old electrochemical cell (see text) at $1.0 \mathrm{~V}$ with $0.1 \%$ formic acid in $50 / 50$ ( vol/vol) acetonitrile/water; flow rate $10 \mu \mathrm{L} / \mathrm{min}$. Middle panel: spectrum with new cell at the same conditions as the top panel. Bottom panel: spectrum obtained with new cell at $1 \mathrm{~V}$ in 5/47.5/47.5 (vol/vol/vol) formic acid/acetonitrile/water; flow rate $2 \mu \mathrm{L} / \mathrm{min}$. Labeled ions: $\mathrm{A}$, non-cleavage oxidation products (mass -2 and $+16 \mathrm{Da}$ ); $\mathrm{B}, \mathrm{N}$-terminal cleavage product (DRVY-2); C, C-terminal cleavage product (IHPFHL); D, dimerized peptide (double mass $-2 \mathrm{Da}$ ). Oxidized background ions, not attributable to angiotensin I, are indicated with an asterisk.

which exhibited much stronger adsorption and sample delay. The increased formic acid concentration makes the solution more acidic, but also increases the electrolyte concentration, reflected in a higher recorded cell current. The separate contributions have to be investigated in more detail to further optimize the reactions in a rational way.

Cleavage yield obtained with the old cell for the proteins RNase I and insulin was well reproducible with the new cell under the new conditions. The cleavage of apomyoglobin took place readily in the old cell, but unfortunately the new cell failed to produce cleavage products. The new conditions were used for all subsequent protein samples described above (Tables 1 and 2).

The longer interaction times with the new cell also necessitated better cleaning procedures. Injection of a loop volume of $5 \%$ formic acid in water, while applying a $1.2 \mathrm{~V}$ potential was generally sufficient to eliminate carry-over of protein oxidation products.

\section{Discussion and Future Prospects}

Electrochemical protein cleavage occurs to varying degrees. All proteins are oxidized to a significant degree judged from both the emergence of peptide peaks and the shifts in the protein peaks. For some proteins, cleavage products were not observed at the current experimental conditions. The large improvements achieved by a few simple optimization steps with the new cell suggest that further optimization should lead to successful cleavage of these proteins too. When a 
larger range of proteins are studied, it will be possible to gain more insight into the reasons for susceptibility to cleavage among different proteins.

The only example described previously of an electrochemically cleaved protein was hen egg white lysozyme, specifically cleaved at the tryptophan-62 residue [8]. Although this tryptophan was part of an unusual WW pair, it was already noted by the authors that specificity of the cleavage reaction to this sequence was unlikely. Other potential cleavage products of lysozyme were missed, possibly because the oxidation products were separated with SDS-PAGE before isolation and N-terminal sequencing. Cleavage at other tryptophan or tyrosine residues than the one reported should give rise to small peptides (less than $5 \mathrm{kD}$ ). We observed cleavage of lysozyme only after breaking the disulfide bonds (Figure 6). Therefore, the presence of disulfide bonds can at least partially account for the fact that some of the tested proteins produced few or no peptides upon oxidation. Protein unfolding and accessibility of oxidizable residues is of prime importance for good protein digestion. The presence of disulfide bonds is undesirable for any digestion method. In addition to interfering with unfolding, some peptides will be internally linked by disulfide bridges, which complicates database searching and MS/MS analysis. To avoid these problems, disulfide bonds are generally reduced and the thiol groups alkylated before proteolytic digestion. This can of course also be done before electrochemical oxidation, although the potential speed advantage of the method will largely be lost. In addition, residues of reagents like iodoacetamide clearly interfere with electrospray ionization of peptides and proteins and may also have an effect on the oxidation reactions themselves.

The presence of disulfide bonds cannot account for all differences in cleavage yields, since both insulin and $\alpha$-lactalbumin contain disulfide bonds, but still exhibit significant protein cleavage. Interestingly, the disulfide group is not always oxidized when present in a peptide formed by cleavage.

The occurrence of tyrosine and tryptophan in proteins in the Swiss-Prot database is less than half that of arginine and lysine (the residues to which trypsin is specific). The average size of the electrochemically generated peptides would be about $2.4 \mathrm{kD}$. The lack of a defined C-terminal basic amino acid is another disadvantage, but this is shared by all methods not specific for basic amino acids.

Chymotrypsin is the only known proteolytic enzyme with partial specificity for tyrosine and tryptophan, but it also cleaves adjacent to phenylalanine and several other amino acid residues (leucine, methionine, histidine): in general amino acids with bulky side chains [1]. This low selectivity produces comparatively small peptides [1], making chymotrypsin less useful for peptide mapping in most cases.

Electrochemistry has some clear advantages over enzymatic and chemical methods. The main advan- tage is the on-line coupling to a mass spectrometer, without the need to remove excess reagents, enzyme, or buffers. The digestion time is very short: of the order of minutes. It also shares some of the disadvantages of chemical methods: reactions with impurities and unwanted reactions with other (sulfur-containing) amino acids. As discussed above, cleaning of the electrode is still an important issue with the current system, but this should be technically addressable. More in-depth study of solvent conditions and electrode type and surface is required to prevent adsorption and at the same time improve cleavage yield. Even at the current state of development, with simple experimental conditions, protein cleavage is specific for $\mathrm{Y}$ and $\mathrm{W}$. Multiple cleavages are achievable and for small proteins complete digestion has already been approached.

\section{Conclusions}

The electrochemical cleavage of proteins is feasible and specific for tyrosine and tryptophan. This indicates the possibility to develop electrochemistry as an instrumental alternative to enzymatic or chemical protein digestion. The clear advantages are the speed and easy coupling of the electrochemical flow cell to MS or LC/MS, and the distinct amino acid specificity. Nevertheless, side reactions of impurities and protein oxidation reactions that do not produce protein backbone cleavage still complicate the resulting oxidation product mixture. Proteins show very different relative yields of cleavage versus non-cleavage products. Further optimization of experimental conditions is critical to develop a generally useful electrochemical digestion method.

\section{Acknowledgments}

The authors thank ESA Inc., Bedford, Masachusetts, U.S.A., for providing the Coulochem III electrochemical detector and the 5021 conditioning cell.

\section{References}

1. Kellner, R.; Lottspeich, F.; Meyer, H. E., Eds.; Microcharacterization of Proteins, 2nd ed; Wiley-VCH: Weinheim, Germany, 1999.

2. Malfoy, B.; Reynaud, J. A. J. Electroanal. Chem. 1980, 114(2), 213-223.

3. Reynaud, J. A.; Malfoy, B.; Canesson, P. J. Electroanal. Chem. 1980, 114(2), 195-211.

4. Permentier, H. P.; Jurva, U.; Barroso, B.; Bruins, A. P. Rapid Commun. Mass Spectrom. 2003, 17, 1585-1592.

5. Iwasaki, H.; Cohen, L. A.; Witkop, B. J. Am. Chem. Soc. 1963, 85, 3701-3702.

6. Farber, L.; Cohen, L. A. Biochemistry 1966, 5(3), 1027-1034.

7. MacDonald, S. M.; Roscoe, S. G. Electrochim. Acta 1997, 42(8), 1189-1200.

8. Walton, D. J.; Richards, P. G.; Heptinstall, J.; Coles, B. Electrochim. Acta 1997, 42(15), 2285-2294. 
9. Schmir, G. L.; Cohen, L. A.; Witkop, B. J. Am. Chem. Soc. 1959, $81,2228-2233$.

10. Zhou, F. M.; Van Berkel, G. J. Anal. Chem. 1995, 67(20), 3643-3649.

11. Jurva, U.; Wikström, H. V.; Bruins, A. P. Rapid Commun. Mass Spectrom. 2000, 14, 529-533.

12. Diehl, G.; Karst, U. Anal. Bioanal. Chem. 2002, 373, 390-398.

13. Jurva, U.; Wikström, H. V.; Weidolf, L.; Bruins, A. P. Rapid Commun. Mass Spectrom. 2003, 17, 800-810.

14. Schey, K. L.; Finley, E. L. Acc. Chem. Res. 2000, 33(5), 299-306.

15. Kendall, G.; Cooper, H. J.; Heptinstall, J.; Derrick, P. J.;
Walton, D. J.; Peterson, I. R. Arch. Biochem. Biophys. 2001, 392(2), 169-179.

16. Maleknia, S. D.; Downard, K. Mass Spectrom. Rev. 2001, 20, 388-401.

17. Sharp J. S.; Becker, J. M.; Hettich, R. L. Anal. Chem. 2004, 76(3), 672-683.

18. Giulivi, C.; Traaseth, N. J.; Davies, K. J. A. Amino Acids 2003, $25,227-232$.

19. Lund, H.; Baizer, M. M., Eds.; Organic Electrochemistry, an Introduction and Guide, 3rd ed.; Marcel Dekker: New York, 1991. 